\title{
Application of the Exp-function method to the $(2+1)$-dimensional Boiti-Leon-Pempinelli equation using symbolic computation
}

\author{
İsmail Aslan* \\ Department of Mathematics, İzmir Institute of Technology, Urla, İzmir 35430, Turkey
}

(Received 23 March 2009; revised version received 30 September 2009; accepted 18 January 2010)

\begin{abstract}
This paper deals with the so-called Exp-function method for studying a particular nonlinear partial differential equation (PDE): the $(2+1)$-dimensional Boiti-Leon-Pempinelli equation. The method is constructive and can be carried out in a computer with the aid of a computer algebra system. The obtained generalized solitary wave solutions contain more arbitrary parameters compared with the earlier works, and thus, they are wider. This means that our method is effective and powerful for constructing exact and explicit analytic solutions to nonlinear PDEs.
\end{abstract}

Keywords: Exp-function method; $(2+1)$-dimensional Boiti-Leon-Pempinelli equation; solitary wave solutions

2000 AMS Subject Classifications: 35Q53; 35Q51; 35C05

\section{Introduction}

Many fundamental problems in nonlinear sciences are described by nonlinear partial differential equations (NPDEs). It is also believed that many problems of future technologies will be described in the same way. Physical problems and the increasing availability of technology (including computer algebra systems such as MATHEMATICA, MAPLE, or MATLAB) have motivated the development of many analytic methods for finding exact and explicit solutions of such equations. Thus, in the last four decades or so, many different methods have been presented in the open literature to look for exact solutions of these equations. To mention some, Darboux transformation [33], Hirota bilinear method [27], inverse scattering transformation [2], symmetry method [9], Weierstrass function method [43], Jacobi elliptic function method [29], Sine-Cosine function [40], Tanh-Coth function [32], F-expansion method [1], homotopy perturbation method $[15,16,23]$, variational iteration method [14,22,38], Adomian decomposition method $[3,17,18]$, $\left(G^{\prime} / G\right)$-expansion method $[4,6,7,35,36,39]$, and so forth. However, most of the methods have some restrictions for application purposes.

*Email: ismailaslan@iyte.edu.tr

ISSN 0020-7160 print/ISSN 1029-0265 online

(C) 2011 Taylor \& Francis

DOI: 10.1080/00207161003706521

http://www.informaworld.com 
Since the nonlinear phenomena is so complex, it is still an open problem to find more powerful techniques to deduce more new exact solutions to the nonlinear equations of modelling problems. Recently, $\mathrm{He}$ and $\mathrm{Wu}$ [25] introduced the so-called Exp-function method, a relatively new method. The Exp-function 'method' consists of trying rational combinations of exponential functions as an 'ansatz' to find exact solutions of the ordinary differential equation (ODE) for travelling waves of the original equation. It can be used as an alternative method for obtaining approximate and analytic solutions of many NPDEs. The Exp-function method was found to be one of the most effective methods to search for exact solutions of NPDEs [5,24,26,34,42]. It has been successfully extended by the authors $[19,45]$ to study some nonlinear equations with variable coefficients. Moreover, being less restrictive and concise, it is modified by the researchers [12,13,46,47] to analyse some special nonlinear differential-difference equations.

In this paper, we consider the following $(2+1)$-dimensional Boiti-Leon-Pempinelli (BLP) equation [8]

$$
\left\{\begin{array}{l}
u_{t y}-\left(u^{2}-u_{x}\right)_{x y}-2 v_{x x x}=0, \\
v_{t}-v_{x x}-2 u v_{x}=0,
\end{array}\right.
$$

and apply the Exp-function method to the BLP equation to construct new and interesting exact solutions. The integrability of Equation (1) was shown in [8]. In [21], it was also observed that Equation (1) was Hamiltonian. Both Boiti et al. and Garagash [8,21] derived Bäcklund transformation for Equation (1). The fact that the sine-Gordon equation or the sinh-Gordon equation can be derived from the BLP equation by a certain transformation was pointed out in [21]. A great deal of research work has been invested in the literature for the study of BLP equation. To mention some, Weiss [41] has shown that the BLP system to be solvable in terms of a commuting set of nonlinear Hamiltonian ordinary differential equations, which are related to the fixed points of the Bäcklund transformation for this system. Yurov [44] studied the Darboux and Laplace transformations for the BLP equation, as well as demonstrated that it can be reduced to the Burgers (and anti-Burgers) equation in a one-dimensional limit. By using a further extended tanh method, Lü and Zhang [31] constructed explicit exact solutions for the BLP equation. Huang and Zhang [28] obtained several types of exact travelling wave solutions to the BLP equation by implementing an improved projective Riccati equation method. Based on the variable separation solution and by selecting appropriate multi-valued functions, Dai and $\mathrm{Ni}$ [10] investigated novel interactions between a special bell-like semi-foldon and a special peakon-like semi-foldon for the BLP equation. Inspired by the extended tanh function method and the direct method of symmetry reduction, Lü [30] presented a Burgers equation-based constructive method for solving NPDEs and had taken the BLP equation as an example to illustrate the algorithm. By giving some types of general solutions of a first-order nonlinear ODE with six degree and presenting a new generalized algebra method to find more exact solutions of NPDEs, Ren et al. [37] obtained many types of solutions for the BLP equation. The extended tanh method has been successfully applied to the BLP equation by Feng et al. [20] to search for exact and explicit solutions. Recently, a new mapping method is used by Dai and Wang [11] to obtain the variable separation solutions (with two arbitrary functions) of the BLP equation, and so forth.

\section{The Exp-function method}

To begin with, suppose that we have a NPDE for $u(x, y, t)$ in the form

$$
P\left(u, u_{t}, u_{x}, u_{y}, u_{t t}, u_{t x}, u_{t y}, u_{x x}, u_{x y}, u_{y y}, \ldots\right)=0,
$$

where $P$ is a polynomial in its arguments. We summarize the algorithm as follows: 
Step 1 By taking $u(x, y, t)=U(\zeta), \zeta=k x+m y+w t$, where $k, m$, and $w$ are arbitrary nonzero constants, look for travelling wave solutions of Equation (2) and transform it to the ODE

$$
Q\left(U, U^{\prime}, U^{\prime \prime}, \ldots\right)=0,
$$

where the prime denotes derivative with respect to $\zeta$.

Step 2 Integrate Equation (3), if possible, term by term one or more times. This yields constant(s) of integration. The integration constant(s) can be set to zero for simplicity.

Step 3 Suppose the solution $U(\zeta)$ of Equation (3) can be expressed in the form

$$
U(\zeta)=\frac{a_{c} \exp (c \zeta)+\cdots+a_{-d} \exp (-d \zeta)}{b_{p} \exp (p \zeta)+\cdots+b_{-q} \exp (-q \zeta)},
$$

where $c, d, p$, and $q$ are unknown positive integers to be determined, $a_{i}$ and $b_{j}$ are unknown constants.

Step 4 Determine the highest order nonlinear term and the linear term of highest order in Equation (3) and express them in terms of Equation (4). Then, in the resulting terms, balance the highest order Exp-function to determine $c$ and $p$, and the lowest order Exp-function to determine $d$ and $q$.

Step 5 Substitute Equation (4) into Equation (3) and equate the coefficients of exp $(n \zeta)$ to zero, and obtain a system of algebraic equations for $a_{i}, b_{j}, k, m$, and $w$. Then, to determine these constants, solve the system with the aid of a computer algebra system.

Step 6 Substitute the values solved in Step 5 into expression (4) and find the travelling wave solutions of Equation (2). Then, it is necessary to substitute them into the original Equation (2) to assure the correctness of the solutions.

\section{Solutions to the BLP equation by the Exp-function method}

To seek for the travelling wave solutions to the BLP equation (1), we first make the transformation $u(x, y, t)=U(\zeta), v(x, y, t)=V(\zeta), \zeta=k x+m y+w t$, where $k, m$, and $w$ are constants to be determined later. Then, we get the system of ODEs

$$
\begin{aligned}
w m U^{\prime \prime} & =k m\left(U^{2}-k U^{\prime}\right)^{\prime \prime}+2 k^{3} V^{\prime \prime \prime}, \\
w V^{\prime} & =k^{2} V^{\prime \prime}+2 k U V^{\prime},
\end{aligned}
$$

where the prime denotes derivative with respect to $\zeta$. By integrating the first equation in Equation (5) twice and setting the constants of integration to zero, we get

$$
V=\frac{m}{2 k} U+\frac{m}{2 k^{3}} \int\left(w U-k U^{2}\right) \mathrm{d} \zeta
$$

Moreover, substituting Equation (6) into the second equation of Equation (5), we obtain

$$
k^{4} U^{\prime \prime}-2 k^{2} U^{3}+3 k w U^{2}-w^{2} U=0 .
$$

Now, we consider the ansatz

$$
U(\zeta)=\frac{a_{c} \exp (c \zeta)+\cdots+a_{-d} \exp (-d \zeta)}{b_{p} \exp (p \zeta)+\cdots+b_{-q} \exp (-q \zeta)}
$$


for the solution of Equation (7) and balance the terms $U^{\prime \prime}$ and $U^{3}$. By a simple calculation, we have

$$
U^{\prime \prime}=\frac{k_{1} \exp [(c+3 p) \zeta]+\cdots}{k_{2} \exp [4 p \zeta]+\cdots}
$$

and

$$
U^{3}=\frac{k_{3} \exp [3 c \zeta]+\cdots}{k_{4} \exp [3 p \zeta]+\cdots}=\frac{k_{3} \exp [(3 c+p) \zeta]+\cdots}{k_{4} \exp [4 p \zeta]+\cdots},
$$

where $k_{i} \mathrm{~s}$ are determined coefficients for simplicity. Balancing highest order of Exp-function in Equations (9) and (10), we have

$$
c+3 p=3 c+p
$$

which leads to the result

$$
p=c \text {. }
$$

Similarly, from the ansatz (8), we have

$$
U^{\prime \prime}=\frac{\cdots+l_{1} \exp [-(d+3 q) \zeta]}{\cdots+l_{2} \exp [-4 q \zeta]}
$$

and

$$
U^{3}=\frac{\cdots+l_{3} \exp [-3 d \zeta]}{\cdots+l_{4} \exp [-3 q \zeta]}=\frac{\cdots+l_{3} \exp [-(3 d+q) \zeta]}{\cdots+l_{4} \exp [-4 q \zeta]}
$$

where $l_{i}$ s are determined coefficients for simplicity. Balancing lowest order of Exp-function in Equations (13) and (14), we have

$$
-(d+3 q)=-(3 d+q)
$$

which leads to the result

$$
q=d
$$

We can freely choose the values of $c$ and $d$ in general. However, the final solution does not strongly depend on the values of $c$ and $d[18,38]$.

Case 1. $p=c=1, d=q=1$.

In this case, the solution of Equation (7) can be expressed as

$$
U(\zeta)=\frac{a_{1} \exp (\zeta)+a_{0}+a_{-1} \exp (-\zeta)}{b_{1} \exp (\zeta)+b_{0}+b_{-1} \exp (-\zeta)}
$$

We take $b_{1}=1$ in Equation (17) for simplicity. Then, substituting Equation (17) into Equation (7), we have

$$
\frac{1}{A}\left[C_{0}+C_{1} \exp (\zeta)+C_{2} \exp (2 \zeta)+C_{3} \exp (3 \zeta)+C_{4} \exp (4 \zeta)+C_{5} \exp (5 \zeta)+C_{6} \exp (6 \zeta)\right]=0
$$


where

$$
\begin{aligned}
A= & \left(\exp (2 \zeta)+b_{-1}+b_{0} \exp (\zeta)\right)^{3}, \\
C_{0}= & -2 k^{2} a_{-1}^{3}+3 k w a_{-1}^{2} b_{-1}-w^{2} a_{-1} b_{-1}^{2}, \\
C_{1}= & -6 k^{2} a_{-1}^{2} a_{0}+6 k w a_{-1} a_{0} b_{-1}+k^{4} a_{0} b_{-1}^{2}-w^{2} a_{0} b_{-1}^{2}+3 k w a_{-1}^{2} b_{0} \\
& -k^{4} a_{-1} b_{-1} b_{0}-2 w^{2} a_{-1} b_{-1} b_{0}, \\
C_{2}= & 3 k w a_{-1}^{2}-6 k^{2} a_{-1} a_{0}^{2}-6 k^{2} a_{-1}^{2} a_{1}-4 k^{4} a_{-1} b_{-1}-2 w^{2} a_{-1} b_{-1}+3 k w a_{0}^{2} b_{-1} \\
& +6 k w a_{-1} a_{1} b_{-1}+4 k^{4} a_{1} b_{-1}^{2}-w^{2} a_{1} b_{-1}^{2}+6 k w a_{-1} a_{0} b_{0}-k^{4} a_{0} b_{-1} b_{0}-2 w^{2} a_{0} b_{-1} b_{0} \\
& +k^{4} a_{-1} b_{0}^{2}-w^{2} a_{-1} b_{0}^{2}, \\
C_{3}= & 6 k w a_{-1} a_{0}-2 k^{2} a_{0}^{3}-12 k^{2} a_{-1} a_{0} a_{1}-6 k^{4} a_{0} b_{-1}-2 w^{2} a_{0} b_{-1}+6 k w a_{0} a_{1} b_{-1} \\
& +3 k^{4} a_{-1} b_{0}-2 w^{2} a_{-1} b_{0}+3 k w a_{0}^{2} b_{0}+6 k w a_{-1} a_{1} b_{0}+3 k^{4} a_{1} b_{-1} b_{0} \\
& -2 w^{2} a_{1} b_{-1} b_{0}-w^{2} a_{0} b_{0}^{2}, \\
C_{4}= & 4 k^{4} a_{-1}-w^{2} a_{-1}+3 k w a_{0}^{2}+6 k w a_{-1} a_{1}-6 k^{2} a_{0}^{2} a_{1}-6 k^{2} a_{-1} a_{1}^{2}-4 k^{4} a_{1} b_{-1} \\
& -2 w^{2} a_{1} b_{-1}+3 k w a_{1}^{2} b_{-1}-k^{4} a_{0} b_{0}-2 w^{2} a_{0} b_{0}+6 k w a_{0} a_{1} b_{0} \\
& +k^{4} a_{1} b_{0}^{2}-w^{2} a_{1} b_{0}^{2}, \\
C_{5}= & k^{4} a_{0}-w^{2} a_{0}+6 k w a_{0} a_{1}-6 k^{2} a_{0} a_{1}^{2}-k^{4} a_{1} b_{0}-2 w^{2} a_{1} b_{0}+3 k w a_{1}^{2} b_{0}, \\
C_{6}= & -w^{2} a_{1}+3 k w a_{1}^{2}-2 k^{2} a_{1}^{3} .
\end{aligned}
$$

Equating the coefficients of $\exp (j \zeta)$ to zero in Equation (18) and solving the resulting algebraic system for $a_{1}, a_{0}, a_{-1}, b_{0}, b_{-1}, k, m$, and $w$, we get the following solution sets (denoted in curly brackets from now on) and the corresponding generalized solitary wave solutions to Equation (1):

Case 1.1

$$
\begin{gathered}
\left\{a_{-1}=0, b_{-1}=\frac{-a_{0}^{2} \mp k a_{0} b_{0}}{k^{2}}, a_{1}=\mp k, w=\mp k^{2}, a_{0}=a_{0}, b_{0}=b_{0}, k=k, m=m\right\}, \\
u_{1}(x, y, t)=\frac{-k^{2} \exp \left(k x+m y-k^{2} t\right)}{a_{0}+k\left(b_{0}+\exp \left(k x+m y-k^{2} t\right)\right)} \\
v_{1}(x, y, t)=-\frac{m}{2}, \\
u_{2}(x, y, t)=\frac{-k^{2} \exp \left(k x+m y+k^{2} t\right)}{a_{0}-k\left(b_{0}+\exp \left(k x+m y+k^{2} t\right)\right)}, \\
v_{2}(x, y, t)=\frac{m}{2}+\frac{m\left(k b_{0}-a_{0}\right)}{a_{0}-k\left(b_{0}+\exp \left(k x+m y+k^{2} t\right)\right)},
\end{gathered}
$$

where $a_{0}, b_{0}, k$, and $m$ are arbitrary constants.

Case 1.2

$$
\left\{a_{-1}=0, a_{1}=0, b_{-1}=0, b_{0}=\mp \frac{a_{0}}{k}, w=\mp k^{2}, a_{0}=a_{0}, k=k, m=m\right\},
$$




$$
\begin{aligned}
& u_{3}(x, y, t)=\frac{k a_{0}}{k \exp \left(k x+m y-k^{2} t\right)-a_{0}}, \\
& v_{3}(x, y, t)=\frac{m a_{0}}{k \exp \left(k x+m y-k^{2} t\right)-a_{0}}, \\
& u_{4}(x, y, t)=\frac{k a_{0}}{k \exp \left(k x+m y+k^{2} t\right)+a_{0}}, \\
& v_{4}(x, y, t)=0,
\end{aligned}
$$

where $a_{0}, k$, and $m$ are arbitrary constants.

Case 1.3

$$
\begin{gathered}
\left\{a_{-1}=\mp 2 k b_{-1}, a_{1}=0, b_{0}=0, a_{0}=0, w=\mp 2 k^{2}, b_{-1}=b_{-1}, k=k, m=m\right\}, \\
u_{5}(x, y, t)=-\frac{2 k b_{-1}}{b_{-1}+\exp \left(2\left(k x+m y-2 k^{2} t\right)\right)}, \\
v_{5}(x, y, t)=-\frac{2 m b_{-1}}{b_{-1}+\exp \left(2\left(k x+m y-2 k^{2} t\right)\right)}, \\
u_{6}(x, y, t)=\frac{2 k b_{-1}}{b_{-1}+\exp \left(2\left(k x+m y+2 k^{2} t\right)\right)}, \\
v_{6}(x, y, t)=0,
\end{gathered}
$$

where $b_{-1}, k$, and $m$ are arbitrary constants.

Case $2 p=c=2, d=q=1$.

Then, the trial function (8) becomes

$$
U(\zeta)=\frac{a_{2} \exp (2 \zeta)+a_{1} \exp (\zeta)+a_{0}+a_{-1} \exp (-\zeta)}{b_{2} \exp (2 \zeta)+b_{1} \exp (\zeta)+b_{0}+b_{-1} \exp (-\zeta)}
$$

We set $b_{2}=1$ for simplicity, and thus, Equation (28) takes the form

$$
U(\zeta)=\frac{a_{2} \exp (2 \zeta)+a_{1} \exp (\zeta)+a_{0}+a_{-1} \exp (-\zeta)}{\exp (2 \zeta)+b_{1} \exp (\zeta)+b_{0}+b_{-1} \exp (-\zeta)}
$$

Substituting Equation (29) into Equation (7), we get the equation

$$
\left(\exp (3 \zeta)+b_{-1}+\exp (\zeta) b_{0}+\exp (2 \zeta) b_{1}\right)^{-3} \sum_{j=0}^{9} C_{j} \exp (j \zeta)=0
$$

We omit to display the coefficients $C_{j}$ explicitly. Then, equating the coefficients of $\exp (j \zeta)$ to zero and solving the resulting algebraic system for $a_{2}, a_{1}, a_{0}, a_{-1}, b_{1}, b_{0}, b_{-1}, k, m$, and $w$, we get the following solution sets and the corresponding generalized solitary wave solutions to Equation (1): 
Case 2.1

$$
\begin{gathered}
\left\{a_{-1}=0, a_{2}=0, b_{-1}=0, a_{1}=\frac{1}{2}\left(\mp k b_{1}-\sqrt{k^{2}\left(b_{1}^{2}-4 b_{0}\right)}\right), a_{0}=\mp k b_{0},\right. \\
\left.w=\mp k^{2}, b_{1}=b_{1}, b_{0}=b_{0}, k=k, m=m\right\}, \\
u_{7}(x, y, t)=\frac{-k b_{0}-\frac{1}{2}\left(k b_{1}+\sqrt{k^{2}\left(b_{1}^{2}-4 b_{0}\right)}\right) \exp \left(k x+m y-k^{2} t\right)}{\exp \left(2\left(k x+m y-k^{2} t\right)\right)+b_{0}+b_{1} \exp \left(k x+m y-k^{2} t\right)}, \\
v_{7}(x, y, t)=-\frac{m\left(2 k b_{0}+\left(k b_{1}+\sqrt{k^{2}\left(b_{1}^{2}-4 b_{0}\right)}\right) \exp \left(k x+m y-k^{2} t\right)\right)}{2 k\left(b_{0}+\left(b_{1}+\exp \left(k x+m y-k^{2} t\right)\right) \exp \left(k x+m y-k^{2} t\right)\right)}, \\
u_{8}(x, y, t)=\frac{k b_{0}+\frac{1}{2}\left(k b_{1}-\sqrt{k^{2}\left(b_{1}^{2}-4 b_{0}\right)}\right) \exp \left(k x+m y+k^{2} t\right)}{\exp \left(2\left(k x+m y+k^{2} t\right)\right)+b_{0}+b_{1} \exp \left(k x+m y+k^{2} t\right)}, \\
v_{8}(x, y, t)=0,
\end{gathered}
$$

where $b_{0}, b_{1}, k$, and $m$ are arbitrary constants.

Case 2.2

$$
\begin{aligned}
& \left\{a_{-1}=0, a_{2}=\mp k, b_{-1}=0, a_{1}=\frac{1}{2}\left(\mp k b_{1}-\sqrt{k^{2}\left(b_{1}^{2}-4 b_{0}\right)}\right), a_{0}=0,\right. \\
& \left.w=\mp k^{2}, b_{1}=b_{1}, b_{0}=b_{0}, k=k, m=m\right\}, \\
& u_{9}(x, y, t)=\frac{-k \exp \left(2\left(k x+m y-k^{2} t\right)\right)-\frac{1}{2}\left(k b_{1}+\sqrt{k^{2}\left(b_{1}^{2}-4 b_{0}\right)}\right) \exp \left(k x+m y-k^{2} t\right)}{\exp \left(2\left(k x+m y-k^{2} t\right)\right)+b_{0}+b_{1} \exp \left(k x+m y-k^{2} t\right)}, \\
& v_{9}(x, y, t)=\frac{-m}{2}, \\
& u_{10}(x, y, t)=\frac{k \exp \left(2\left(k x+m y+k^{2} t\right)\right)+\frac{1}{2}\left(k b_{1}-\sqrt{k^{2}\left(b_{1}^{2}-4 b_{0}\right)}\right) \exp \left(k x+m y+k^{2} t\right)}{\exp \left(2\left(k x+m y+k^{2} t\right)\right)+b_{0}+b_{1} \exp \left(k x+m y+k^{2} t\right)}, \\
& v_{10}(x, y, t)=-\frac{m\left(k b_{0}+\left(\sqrt{k^{2}\left(b_{1}^{2}-4 b_{0}\right)}-k \exp \left(k x+m y+k^{2} t\right)\right) \exp \left(k x+m y+k^{2} t\right)\right)}{2 k\left(b_{0}+\left(b_{1}+\exp \left(k x+m y+k^{2} t\right)\right) \exp \left(k x+m y+k^{2} t\right)\right)},
\end{aligned}
$$

where $b_{0}, b_{1}, k$, and $m$ are arbitrary constants.

\section{Case 2.3}

$$
\begin{aligned}
& \left\{a_{-1}=0, a_{2}=\mp 2 k, b_{-1}=0, a_{1}=0, a_{0}=0, b_{1}=0, w=\mp 2 k^{2}, b_{0}=b_{0}, k=k, m=m\right\} \\
& u_{11}(x, y, t)=-\frac{2 k \exp \left(2\left(k x+m y-2 k^{2} t\right)\right)}{b_{0}+\exp \left(2\left(k x+m y-2 k^{2} t\right)\right)}
\end{aligned}
$$




$$
\begin{aligned}
& v_{11}(x, y, t)=-m, \\
& u_{12}(x, y, t)=\frac{2 k \exp \left(2\left(k x+m y+2 k^{2} t\right)\right)}{b_{0}+\exp \left(2\left(k x+m y+2 k^{2} t\right)\right)}, \\
& v_{12}(x, y, t)=m-\frac{2 m b_{0}}{b_{0}+\exp \left(2\left(k x+m y+2 k^{2} t\right)\right)},
\end{aligned}
$$

where $b_{0}, k$, and $m$ are arbitrary constants.

Case 2.4

$$
\begin{aligned}
& \left\{a_{-1}=\mp 3 k b_{-1}, a_{2}=0, a_{1}=0, a_{0}=0, b_{1}=0, b_{0}=0, w=\mp 3 k^{2}, b_{-1}=b_{-1},\right. \\
& k=k, m=m\}, \\
& u_{13}(x, y, t)=-\frac{3 k b_{-1}}{b_{-1}+\exp \left(3\left(k x+m y-3 k^{2} t\right)\right)}, \\
& v_{13}(x, y, t)=-\frac{3 m b_{-1}}{b_{-1}+\exp \left(3\left(k x+m y-3 k^{2} t\right)\right)}, \\
& u_{14}(x, y, t)=\frac{3 k b_{-1}}{b_{-1}+\exp \left(3\left(k x+m y+3 k^{2} t\right)\right)}, \\
& v_{14}(x, y, t)=0,
\end{aligned}
$$

where $b_{-1}, k$, and $m$ are arbitrary constants.

Case 2.5

$$
\begin{gathered}
\left\{a_{-1}=0, a_{2}=\mp 3 k, b_{-1}=0, a_{1}=\mp 3 k b_{1}, a_{0}=\mp 3 k b_{0}, w=\mp 3 k^{2}, b_{1}=b_{1},\right. \\
\left.b_{0}=b_{0}, k=k, m=m\right\}, \\
u_{15,16}(x, y, t)=\frac{\mp 3 k \exp \left(2\left(k x+m y \mp 3 k^{2} t\right)\right) \mp 3 k b_{0} \mp 3 k b_{1} \exp \left(k x+m y \mp 3 k^{2} t\right)}{\exp \left(2\left(k x+m y \mp 3 k^{2} t\right)\right)+b_{0}+b_{1} \exp \left(k x+m y \mp 3 k^{2} t\right)} \\
v_{15,16}(x, y, t)=\mp \frac{3 m}{2},
\end{gathered}
$$

where $b_{0}, b_{1}, k$, and $m$ are arbitrary constants.

Case 2.6

$$
\begin{aligned}
& \left\{a_{-1}=0, a_{2}=\mp 3 k, a_{1}=0, a_{0}=0, b_{1}=0, b_{0}=0, w=\mp 3 k^{2}, b_{-1}\right. \\
& \left.=b_{-1}, k=k, m=m\right\} \text {, } \\
& u_{17}(x, y, t)=\frac{-3 k \exp \left(3\left(k x+m y-3 k^{2} t\right)\right)}{b_{-1}+\exp \left(3\left(k x+m y-3 k^{2} t\right)\right)}, \\
& v_{17}(x, y, t)=\frac{-3 m}{2}, \\
& u_{18}(x, y, t)=\frac{3 k \exp \left(3\left(k x+m y+3 k^{2} t\right)\right)}{b_{-1}+\exp \left(3\left(k x+m y+3 k^{2} t\right)\right)}, \\
& v_{18}(x, y, t)=\frac{3 m}{2}-\frac{3 m b_{-1}}{b_{-1}+\exp \left(3\left(k x+m y+3 k^{2} t\right)\right)},
\end{aligned}
$$

where $b_{-1}, k$, and $m$ are arbitrary constants. 
Case $3 p=c=2, d=q=2$.

Then, the trial function (8) becomes

$$
U(\zeta)=\frac{a_{2} \exp (2 \zeta)+a_{1} \exp (\zeta)+a_{0}+a_{-1} \exp (-\zeta)+a_{-2} \exp (-2 \zeta)}{b_{2} \exp (2 \zeta)+b_{1} \exp (\zeta)+b_{0}+b_{-1} \exp (-\zeta)+b_{-2} \exp (-2 \zeta)}
$$

There are some free parameters in Equation (48), so we set $b_{2}=1, b_{1}=0$, and $b_{-1}=0$ for simplicity, and thus, Equation (48) takes the form

$$
U(\zeta)=\frac{a_{2} \exp (2 \zeta)+a_{1} \exp (\zeta)+a_{0}+a_{-1} \exp (-\zeta)+a_{-2} \exp (-2 \zeta)}{\exp (2 \zeta)+b_{0}+b_{-2} \exp (-2 \zeta)}
$$

By the same manipulations as in the previous cases, we obtain the following solution sets and the corresponding generalized solitary wave solutions to Equation (1).

Case 3.1

$$
\begin{gathered}
\left\{a_{-2}=\mp 2 k b_{-2}, a_{2}=0, a_{-1}=0, a_{1}=0, a_{0}=\mp k b_{0}-\sqrt{k^{2}\left(b_{0}^{2}-4 b_{-2}\right)},\right. \\
\left.w=\mp 2 k^{2}, b_{-2}=b_{-2}, b_{0}=b_{0}, k=k, m=m\right\}, \\
u_{19}(x, y, t)=\frac{-2 k b_{-2} \exp \left(-2\left(k x+m y-2 k^{2} t\right)\right)-k b_{0}-\sqrt{k^{2}\left(b_{0}^{2}-4 b_{-2}\right)}}{b_{0}+\exp \left(2\left(k x+m y-2 k^{2} t\right)\right)+b_{-2} \exp \left(-2\left(k x+m y-2 k^{2} t\right)\right)}, \\
v_{19}(x, y, t)=-\frac{m\left(2 k b_{-2}+\left(k b_{0}+\sqrt{k^{2}\left(b_{0}^{2}-4 b_{-2}\right)}\right) \exp \left(2\left(k x+m y-2 k^{2} t\right)\right)\right)}{k\left(\exp \left(4\left(k x+m y-2 k^{2} t\right)\right)+b_{-2}+b_{0} \exp \left(2\left(k x+m y-2 k^{2} t\right)\right)\right)}, \\
u_{20}(x, y, t)=\frac{2 k b_{-2} \exp \left(-2\left(k x+m y+2 k^{2} t\right)\right)+k b_{0}-\sqrt{k^{2}\left(b_{0}^{2}-4 b_{-2}\right)}}{\exp \left(2\left(k x+m y+2 k^{2} t\right)\right)+b_{-2} \exp \left(-2\left(k x+m y+2 k^{2} t\right)\right)+b_{0}}, \\
v_{20}(x, y, t)=0,
\end{gathered}
$$

where $b_{0}, b_{-2}, k$, and $m$ are arbitrary constants.

\section{Case 3.2}

$$
\begin{aligned}
& \left\{a_{-2}=0, a_{2}=\mp 2 k, a_{-1}=0, a_{1}=0, a_{0}=\mp k b_{0}-\sqrt{-4 k^{2} b_{-2}+k^{2} b_{0}^{2}}, w=\mp 2 k^{2},\right. \\
& \left.b_{-2}=b_{-2}, b_{0}=b_{0}, k=k, m=m\right\}, \\
& u_{21}(x, y, t)=\frac{-2 k \exp \left(2\left(k x+m y-2 k^{2} t\right)\right)-k b_{0}-\sqrt{k^{2}\left(b_{0}^{2}-4 b_{-2}\right)}}{b_{0}+\exp \left(2\left(k x+m y-2 k^{2} t\right)\right)+b_{-2} \exp \left(-2\left(k x+m y-2 k^{2} t\right)\right)}, \\
& v_{21}(x, y, t)=-m, \\
& u_{22}(x, y, t)=\frac{2 k \exp \left(2\left(k x+m y+2 k^{2} t\right)\right)+k b_{0}-\sqrt{k^{2}\left(b_{0}^{2}-4 b_{-2}\right)}}{b_{0}+\exp \left(2\left(k x+m y+2 k^{2} t\right)\right)+b_{-2} \exp \left(-2\left(k x+m y+2 k^{2} t\right)\right)},
\end{aligned}
$$




$$
v_{22}(x, y, t)=-\frac{m\left(\begin{array}{c}
-k \exp \left(4\left(k x+m y+2 k^{2} t\right)\right)+k b_{-2}+\sqrt{k^{2}\left(b_{0}^{2}-4 b_{-2}\right)} \\
\exp \left(2\left(k x+m y+2 k^{2} t\right)\right)
\end{array}\right)}{k\left(\exp \left(4\left(k x+m y+2 k^{2} t\right)\right)+b_{-2}+b_{0} \exp \left(2\left(k x+m y+2 k^{2} t\right)\right)\right)},
$$

where $b_{0}, b_{-2}, k$, and $m$ are arbitrary constants.

Case 3.3

$$
\begin{aligned}
& \left\{a_{-2}=\mp 4 k b_{-2}, a_{2}=0, a_{-1}=0, a_{1}=0, a_{0}=0, b_{0}=0, w=\mp 4 k^{2},\right. \\
& \left.b_{-2}=b_{-2}, k=k, m=m\right\} \\
& u_{23}(x, y, t)=-\frac{4 k b_{-2}}{b_{-2}+\exp \left(4\left(k x+m y-4 k^{2} t\right)\right)}, \\
& v_{23}(x, y, t)=-\frac{4 m b_{-2}}{b_{-2}+\exp \left(4\left(k x+m y-4 k^{2} t\right)\right)}, \\
& u_{24}(x, y, t)=\frac{4 k b_{-2}}{b_{-2}+\exp \left(4\left(k x+m y+4 k^{2} t\right)\right)}, \\
& v_{24}(x, y, t)=0
\end{aligned}
$$

where $b_{-2}, k$, and $m$ are arbitrary constants.

Case 3.4

$$
\begin{aligned}
& \left\{a_{-2}=0, a_{2}=\mp 4 k, a_{-1}=0, a_{1}=0, a_{0}=0, b_{0}=0, w=\mp 4 k^{2},\right. \\
& \left.\quad b_{-2}=b_{-2}, k=k, m=m\right\}, \\
& u_{25}(x, y, t)=-\frac{4 k}{1+b_{-2} \exp \left(-4\left(k x+m y-4 k^{2} t\right)\right)}, \\
& v_{25}(x, y, t)=-2 m, \\
& u_{26}(x, y, t)=\frac{4 k}{1+b_{-2} \exp \left(-4\left(k x+m y+4 k^{2} t\right)\right)}, \\
& v_{26}(x, y, t)=2 m-\frac{4 m b_{-2}}{b_{-2}+\exp \left(4\left(k x+m y+4 k^{2} t\right)\right)},
\end{aligned}
$$

where $b_{-2}, k$, and $m$ are arbitrary constants.

Case $4 \quad p=c=3, d=q=2$.

Then, the trial function (8) becomes

$$
V(\zeta)=\frac{a_{3} \exp (3 \zeta)+a_{2} \exp (2 \zeta)+a_{1} \exp (\zeta)+a_{0}+a_{-1} \exp (-\zeta)+a_{-2} \exp (-2 \zeta)}{b_{3} \exp (3 \zeta)+b_{2} \exp (2 \zeta)+b_{1} \exp (\zeta)+b_{0}+b_{-1} \exp (-\zeta)+b_{-2} \exp (-2 \zeta)}
$$

There are some free parameters in Equation (62), so we set $b_{3}=1, b_{2}=0, b_{1}=0$, and $b_{-1}=0$ for simplicity, and thus Equation (62) takes the form

$$
V(\zeta)=\frac{a_{3} \exp (3 \zeta)+a_{2} \exp (2 \zeta)+a_{1} \exp (\zeta)+a_{0}+a_{-1} \exp (-\zeta)+a_{-2} \exp (-2 \zeta)}{\exp (3 \zeta)+b_{0}+b_{-2} \exp (-2 \zeta)} .
$$

By the same procedure, we obtain the following solution sets and the corresponding generalized solitary wave solutions to Equation (1). 
Case 4.1

$$
\begin{aligned}
& \left\{a_{-2}=0, a_{3}=\mp 5 k, a_{-1}=0, a_{2}=0, a_{0}=0, a_{1}=0, b_{0}=0, w=\mp 5 k^{2},\right. \\
& \left.b_{-2}=b_{-2}, k=k, m=m\right\} \text {, } \\
& u_{27}(x, y, t)=-\frac{5 k}{1+b_{-2} \exp \left(-5\left(k x+m y-5 k^{2} t\right)\right)} \text {, } \\
& v_{27}(x, y, t)=-\frac{5 m}{2}, \\
& u_{28}(x, y, t)=\frac{5 k}{1+b_{-2} \exp \left(-5\left(k x+m y+5 k^{2} t\right)\right)}, \\
& v_{28}(x, y, t)=\frac{5 m}{2}-\frac{5 m b_{-2}}{b_{-2}+\exp \left(5\left(k x+m y+5 k^{2} t\right)\right)} \text {, }
\end{aligned}
$$

where $b_{-2}, k$, and $m$ are arbitrary constants.

Case 4.2

$$
\begin{aligned}
& \left\{a_{-2}=\mp 5 k b_{-2}, a_{3}=0, a_{-1}=0, a_{2}=0, a_{0}=0, a_{1}=0, b_{0}=0, w=\mp 5 k^{2},\right. \\
& \left.\quad b_{-2}=b_{-2}, k=k, m=m\right\}, \\
& u_{29}(x, y, t)=-\frac{5 k b_{-2}}{b_{-2}+\exp \left(5\left(k x+m y-5 k^{2} t\right)\right)}, \\
& v_{29}(x, y, t)=-\frac{5 m b_{-2}}{b_{-2}+\exp \left(5\left(k x+m y-5 k^{2} t\right)\right)}, \\
& u_{30}(x, y, t)=\frac{5 k b_{-2}}{b_{-2}+\exp \left(5\left(k x+m y+5 k^{2} t\right)\right)}, \\
& v_{30}(x, y, t)=0,
\end{aligned}
$$

where $b_{-2}, k$, and $m$ are arbitrary constants.

Case $5 \quad p=c=3, d=q=3$.

Then, the trial function (8) becomes

$$
U(\zeta)=\frac{\begin{array}{c}
a_{3} \exp (3 \zeta)+ \\
+a_{2} \exp (2 \zeta)+a_{1} \exp (\zeta)+a_{0}+a_{-1} \exp (-\zeta) \\
b_{3} \exp (3 \zeta)+a_{-2} \exp (-2 \zeta)+a_{-3} \exp (2 \zeta)+b_{1} \exp (\zeta)+b_{0}+b_{-1} \exp (-\zeta) \\
+b_{-2} \exp (-2 \zeta)+b_{-3} \exp (-3 \zeta)
\end{array}}{.}
$$

There are some free parameters in Equation (70), so we set $b_{3}=1, b_{2}=0, b_{1}=0, b_{-2}=0$, and $b_{-1}=0$ for simplicity, and thus, Equation (70) takes the form

$$
U(\zeta)=\frac{\begin{array}{c}
a_{3} \exp (3 \zeta)+a_{2} \exp (2 \zeta)+a_{1} \exp (\zeta)+a_{0}+a_{-1} \exp (-\zeta) \\
+a_{-2} \exp (-2 \zeta)+a_{-3} \exp (-3 \zeta)
\end{array}}{\exp (3 \zeta)+b_{0}+b_{-3} \exp (-3 \zeta)} .
$$

By the same procedure, we obtain the following solution sets and the corresponding generalized solitary wave solutions to Equation (1). 
Case 5.1

$$
\begin{gathered}
\left\{\begin{array}{c}
a_{-3}=0, a_{3}=\mp 3 k, a_{-2}=0, a_{2}=0, a_{0}=\frac{3}{2}\left(\mp k b_{0}-\sqrt{k^{2}\left(b_{0}^{2}-4 b_{-3}\right)}\right), \\
\left.a_{1}=0, a_{-1}=0, w=\mp 3 k^{2}, b_{-3}=b_{-3}, b_{0}=b_{0}, k=k, m=m\right\} \\
u_{31}(x, y, t)=\frac{-3 k \exp \left(3\left(k x+m y-3 k^{2} t\right)\right)-\frac{3}{2}\left(k b_{0}+\sqrt{k^{2}\left(b_{0}^{2}-4 b_{-3}\right)}\right)}{b_{0}+\exp \left(3\left(k x+m y-3 k^{2} t\right)\right)+b_{-3} \exp \left(-3\left(k x+m y-3 k^{2} t\right)\right)}, \\
v_{31}(x, y, t)=-\frac{3 m}{2}, \\
u_{32}(x, y, t)=\frac{3 k \exp \left(3\left(k x+m y+3 k^{2} t\right)\right)+\frac{3}{2}\left(k b_{0}-\sqrt{k^{2}\left(b_{0}^{2}-4 b_{-3}\right)}\right)}{b_{0}+\exp \left(3\left(k x+m y+3 k^{2} t\right)\right)+b_{-3} \exp \left(-3\left(k x+m y+3 k^{2} t\right)\right)} \\
v_{32}(x, y, t)=-\frac{3 m\left(-k \exp \left(6\left(k x+m y+3 k^{2} t\right)\right)+k b_{-3}+\sqrt{k^{2}\left(b_{0}^{2}-4 b_{-3}\right)}\right)}{2 k\left(\exp \left(6\left(k x+m y+3 k^{2} t\right)\right)+b_{-3}+b_{0} \exp \left(3\left(k x+m y+3 k^{2} t\right)\right)\right)}, \\
\exp \left(3\left(k x+m y+3 k^{2} t\right)\right)
\end{array}\right.
\end{gathered}
$$

where $b_{0}, b_{-3}, k$, and $m$ are arbitrary constants.

\section{Case 5.2}

$$
\begin{aligned}
& \left\{a_{-3}=\mp 3 k b_{-3}, a_{3}=0, a_{-2}=0, a_{2}=0, a_{0}=\frac{3}{2}\left(\mp k b_{0}-\sqrt{k^{2}\left(b_{0}^{2}-4 b_{-3}\right)}\right),\right. \\
& \left.a_{1}=0, a_{-1}=0, w=\mp 3 k^{2}, b_{-3}=b_{-3}, b_{0}=b_{0}, k=k, m=m\right\}, \\
& u_{33}(x, y, t)=\frac{-3 k b_{-3} \exp \left(-3\left(k x+m y-3 k^{2} t\right)\right)-\frac{3}{2}\left(k b_{0}+\sqrt{k^{2}\left(b_{0}^{2}-4 b_{-3}\right)}\right)}{b_{0}+\exp \left(3\left(k x+m y-3 k^{2} t\right)\right)+b_{-3} \exp \left(-3\left(k x+m y-3 k^{2} t\right)\right)}, \\
& v_{33}(x, y, t)=-\frac{3 m\left(2 k b_{-3}+\left(k b_{0}+\sqrt{k^{2}\left(b_{0}^{2}-4 b_{-3}\right)}\right) \exp \left(3\left(k x+m y-3 k^{2} t\right)\right)\right)}{2 k\left(\exp \left(6\left(k x+m y-3 k^{2} t\right)\right)+b_{-3}+b_{0} \exp \left(3\left(k x+m y-3 k^{2} t\right)\right)\right)}, \\
& u_{33}(x, y, t)=\frac{3 k b_{-3} \exp \left(-3\left(k x+m y+3 k^{2} t\right)\right)+\frac{3}{2}\left(k b_{0}-\sqrt{k^{2}\left(b_{0}^{2}-4 b_{-3}\right)}\right)}{b_{0}+\exp \left(3\left(k x+m y+3 k^{2} t\right)\right)+b_{-3} \exp \left(-3\left(k x+m y+3 k^{2} t\right)\right)}, \\
& v_{33}(x, y, t)=0 \text {, }
\end{aligned}
$$

where $b_{0}, b_{-3}, k$, and $m$ are arbitrary constants.

\section{Case 5.3}

$$
\begin{aligned}
& \left\{a_{-3}=0, a_{3}=\mp 6 k, a_{-2}=0, a_{2}=0, a_{0}=0, a_{1}=0, a_{-1}=0, b_{0}=0, w=\mp 6 k^{2},\right. \\
& \left.\quad b_{-3}=b_{-3}, k=k, m=m\right\},
\end{aligned}
$$




$$
\begin{aligned}
& u_{34}(x, y, t)=-\frac{6 k}{1+b_{-3} \exp \left(-6\left(k x+m y-6 k^{2} t\right)\right)}, \\
& v_{34}(x, y, t)=-3 m \\
& u_{35}(x, y, t)=\frac{6 k}{1+b_{-3} \exp \left(-6\left(k x+m y+6 k^{2} t\right)\right)}, \\
& v_{35}(x, y, t)=3 m-\frac{6 m b_{-3}}{b_{-3}+\exp \left(6\left(k x+m y+6 k^{2} t\right)\right)},
\end{aligned}
$$

where $b_{-3}, k$, and $m$ are arbitrary constants.

\section{Case 5.4}

$$
\begin{aligned}
& \left\{a_{-3}=\mp 6 k b_{-3}, a_{3}=0, a_{-2}=0, a_{2}=0, a_{0}=0, a_{1}=0, a_{-1}=0, b_{0}=0, w=\mp 6 k^{2},\right. \\
& \left.\quad b_{-3}=b_{-3}, k=k, m=m\right\}, \\
& u_{36}(x, y, t)=-\frac{6 k b_{-3}}{b_{-3}+\exp \left(6\left(k x+m y-6 k^{2} t\right)\right)}, \\
& v_{36}(x, y, t)=-\frac{6 m b_{-3}}{b_{-3}+\exp \left(6\left(k x+m y-6 k^{2} t\right)\right)}, \\
& u_{37}(x, y, t)=\frac{6 k b_{-3}}{b_{-3}+\exp \left(6\left(k x+m y+6 k^{2} t\right)\right)}, \\
& v_{37}(x, y, t)=0,
\end{aligned}
$$

where $b_{-3}, k$, and $m$ are arbitrary constants.

Remark 1 It is important that one should be aware of the limitations of each of the existing methods and there is no guarantee that they will succeed for a specific nonlinear problem. Any of these techniques can have some advantages and disadvantages. The Exp-function method provides exponential function solutions in a neat form from which we can construct solitary and periodic wave solutions by setting the parameters as special values. It is entirely algorithmic and involves a large amount of tedious calculations that can become virtually unmanageable if attempted manually. However, it does not require a large amount of run-time with the aid of a computer algebra system. The Exp-function method, by assuming the solution of the equation in exponential form with many parameters, entails the solution of several sets of nonlinear algebraic equations that sometimes constitute inconsistent systems.

\section{Conclusion}

Thanks to the improvement in analytic techniques via the rapid advance in computer technology, most of the PDEs arising from applications that were previously intractable can now be routinely solved. In this work, we used the Exp-function method to solve the (2+1)-dimensional BLP equation. Some of the obtained generalized solitary wave solutions have known physical understandings, while others require further insight into their hidden meanings. We verified the obtained solutions by putting them back into the original Equation (1) with the aid of MATHEMATICA; it provides an extra measure of confidence in the results. We predict that the Exp-function method can be found widely applicable in searching for exact and explicit solutions of nonlinear differential equations. 


\section{References}

[1] M.A. Abdou, The extended F-expansion method and its application for a class of nonlinear evolution equations, Chaos Solitons Fractals 31 (2007), pp. 95-104.

[2] M.J. Ablowitz and P.A. Clarkson, Solitons, Nonlinear Evolution Equations and Inverse Scattering, Cambridge University Press, Cambridge, 1991.

[3] G. Adomian, Solving Frontier Problems of Physics: The Decomposition Method, Kluwer, Boston, MA, 1994.

[4] İ. Aslan, Exact and explicit solutions to some nonlinear evolution equations by utilizing the $\left(G^{\prime} / G\right)$-expansion method, Appl. Math. Comput. 215 (2009), pp. 857-863.

[5] İ. Aslan, Generalized solitary and periodic wave solutions to a $(2+1)$-dimensional Zakharov-Kuznetsov equation, Appl. Math. Comput. 217(4) (2010), pp. 1421-1429.

[6] İ. Aslan and T. Öziş, Analytic study on two nonlinear evolution equations by using the $\left(G^{\prime} / G\right)$-expansion method, Appl. Math. Comput. 209 (2009), pp. 425-429.

[7] İ. Aslan and T. Öziş, On the validity and reliability of the $\left(G^{\prime} / G\right)$-expansion method by using higher-order nonlinear equations, Appl. Math. Comput. 211 (2009), pp. 531-536.

[8] M. Boiti, J.J.P. Leon, and F. Pempinelli, Integrable two-dimensional generalization of the sine- and sinh-Gordon equations, Inverse Prob. 3 (1987), pp. 37-49.

[9] G.W. Bluman and S. Kumei, Symmetries and Differential Equations, Springer, Berlin, 1989.

[10] C.Q. Dai and Y.Z. Ni, Novel interactions between semi-foldons of the $(2+1)$-dimensional Boiti-Leon-Pempinelli equation, Phys. Scr. 74 (2006), pp. 584-590.

[11] C. Dai and Y. Wang, Periodic structures based on variable separation solution of the $(2+1)$-dimensional BoitiLeon-Pempinelli equation, Chaos Solitons Fractals 39 (2009), pp. 350-355.

[12] C. Dai, X. Cen, and S. Wu, Exact travelling wave solutions of the discrete sine-Gordon equation obtained via the exp-function method, Nonlinear Anal. 70 (2009), pp. 58-63.

[13] C. Dai, X. Cen, and S. Wu, The application of He's exp-function method to a nonlinear differential-difference equation, Chaos Solitons Fractal 41 (2009), pp. 511-515.

[14] M. Dehghan and A. Saadatmandi, Variational iteration method for solving the wave equation subject to an integral conservation condition, Chaos Solitons Fractals 41 (2009), pp. 1448-1453.

[15] M. Dehghan and F. Shakeri, Use of He's homotopy perturbation method for solving a partial differential equation arising in modeling of flow in porous media, J. Porous Media 11 (2008), pp. 765-778.

[16] M. Dehghan and F. Shakeri, Solution of an integro-differential equation arising in oscillating magnetic fields using He's homotopy perturbation method, Prog. Electromagn. Res. 78 (2008), pp. 361-376.

[17] M. Dehghan and F. Shakeri, The use of the decomposition procedure of Adomian for solving a delay differential equation arising in electrodynamics, Phys. Scr. 78 (2008), pp. 1-11.

[18] M. Dehghan, M. Shakourifar, and A. Hamidi, The solution of linear and nonlinear systems of Volterra functional equations using Adomian-Pade technique, Chaos Solitons Fractals 39 (2009), pp. 2509-2521.

[19] S.A. El-Wakil, M.A. Madkour, and M.A. Abdou, Application of Exp-function method for nonlinear evolution equations with variable coefficients, Phys. Lett. A. 369 (2007), pp. 62-69.

[20] W.G. Feng, K.M. Li, Y.Z. Li, and C. Lin, Explicit exact solutions for $(2+1)$-dimensional Boiti-Leon-Pempinelli equation, Commun. Nonlinear Sci. Numer. Simul. 14 (2009), pp. 2013-2017.

[21] T.I. Garagash, On a modification of the Painlevé test for systems of nonlinear partial differential equations, Theoret. Math. Phys. 100 (1994), pp. 1075-1081.

[22] J.H. He, A new approach to nonlinear partial differential equations, Commun. Nonlinear Sci. Numer. Simul. 2 (1997), pp. 230-235.

[23] J.H. He, An approximate solution technique depending on an artificial parameter: A special example, Commun. Nonlinear Sci. Numer. Simul. 3 (1998), pp. 92-97.

[24] J.H. He and M.A. Abdou, New periodic solutions for nonlinear evolution equations using Exp-function method, Chaos Solitons Fractals 34 (2007), pp. 1421-1429.

[25] J.H. He and X.H. Wu, Exp-function method for nonlinear wave equations, Chaos Solitons Fractals 30 (2006), pp. 700-708.

[26] J.H. He and L.N. Zhang, Generalized solitary solution and compacton-like solution of the Jaulent-Miodek equations using the Exp-function method, Phys. Lett. A. 372 (2008), pp. 1044-1047.

[27] R. Hirota, The Direct Method in Soliton Theory, Cambridge University Press, Cambridge, 1971.

[28] D.J. Huang and H.Q. Zhang, Exact traveling wave solutions for the Boiti-Leon-Pempinelli equation, Chaos Solitons Fractals 22 (2004), pp. 243-247.

[29] G.T. Liu and T.Y. Fan, New applications of developed Jacobi elliptic function expansion methods, Phys. Lett. A 345 (2005), pp. 161-166.

[30] Z. Lü, A Burgers equation-based constructive method for solving nonlinear evolution equations, Phys. Lett. A 353 (2006), pp. 158-160.

[31] Z. Lü and H. Zhang, Soliton like and multi-soliton like solutions for the Boiti-Leon-Pempinelli equation, Chaos Solitons Fractals 19 (2004), pp. 527-531.

[32] W. Malfliet and W. Hereman, The tanh method I: Exact solutions of nonlinear evolution and wave equations, Phys. Scr. 54 (1996), pp. 563-568.

[33] V.B. Matveev and M.A. Salle, Darboux Transformations and Solitons, Springer, Berlin, 1991. 
[34] T. Öziş and İ. Aslan, Exact and explicit solutions to the $(3+1)$-dimensional Jimbo-Miwa equation via the Expfunction method, Phys. Lett. A 372 (2008), pp. 7011-7015.

[35] T. Öziş and İ. Aslan, Symbolic computation and construction of new exact traveling wave solutions to FitzhughNagumo and Klein-Gordon equations, Z. Naturforsch. 64a (2009), pp. 15-20.

[36] T. Öziş and İ. Aslan, Symbolic computations and exact and explicit solutions of some nonlinear evolution equations in mathematical physics, Commun. Theor. Phys. 51 (2009), pp. 577-580.

[37] Y.J. Ren, S.T. Liu, and H.Q. Zhang, A new generalized algebra method and its application in the $(2+1)$ dimensional Boiti-Leon-Pempinelli equation, Chaos Solitons Fractals 32 (2007), pp. 1655-1665.

[38] F. Shakeri and M. Dehghan, Solution of a model describing biological species living together using the variational iteration method, Math. Comput. Model. 48 (2008), pp. 685-699.

[39] M. Wang, X. Li, and J. Zhang, The $\left(G^{\prime} / G\right)$-expansion method and travelling wave solutions of nonlinear evolution equations in mathematical physics, Phys. Lett. A 372 (2008), pp. 417-423.

[40] A.M. Wazwaz, Distinct variants of the KdV equation with compact and noncompact structures, Appl. Math. Comput. 150 (2004), pp. 365-377.

[41] J. Weiss, Factorization of the $(2+1)$-dimensional BLP integrable system by the periodic fixed points of its Bäcklund transformations, Phys. Lett. A 160 (1991), pp. 161-165.

[42] X.H. Wu and J.H. He, Solitary solutions, periodic solutions and compacton-like solutions using the Exp-function method, Comput. Math. Appl. 54 (2007), pp. 966-986.

[43] Z.Y. Yan, An improved algebra method and its applications in nonlinear wave equations, Chaos Solitons Fractals 21 (2004), pp. 1013-1021.

[44] A.V. Yurov, BLP dissipative structures in plane, Phys. Lett. A 262 (1999), pp. 445-452.

[45] S. Zhang, Application of Exp-function method to a KdV equation with variable coefficients, Phys. Lett. A 365 (2007), pp. 448-453.

[46] S. Zhang, Exp-function method for constructing explicit and exact solutions of a lattice equation, Appl. Math. Comput. 199 (2008), pp. 242-249.

[47] S.D. Zhu, Discrete (2+1)-dimensional Toda lattice equation via Exp-function method, Phys. Lett. A 372 (2008), pp. 654-657. 